Open Access

\title{
Three cases of appendicitis with anorexia nervosa under inpatient care
}

\author{
Shu Takakura", Hiroaki Yokoyama', Chie Suzuyama', Keita Tatsushima², Makoto Yamashita', Motoharu Gondou', \\ Chihiro Morita ${ }^{1}$, Tomokazu Hata ${ }^{2}$, Masato Takii ${ }^{1}$, Keisuke Kawai ${ }^{1}$ and Nobuyuki Sudo ${ }^{1,2}$
}

\begin{abstract}
Background: Little is known about the occurrence of appendicitis during the re-nourishment period in anorexia nervosa (AN). We report three cases of appendicitis in patients with AN that occurred after hospitalization for treatment of AN.

Case presentation: Case 1 is a 34-year-old female, case 2 is a 17-year-old female and case 3 is a 38-year-old female. Constipation was observed in all three cases. Careful management of defecation might be essential to prevent appendicitis among AN patients during the re-nourishment period under inpatient care. In addition, mild and diffuse symptoms were observed in all three cases. Therefore, diagnosis proved to be difficult to make and abdominal computed tomography was particularly helpful in all cases. As the symptoms were diffuse, the condition of appendicitis turned out to be more severe and complicated in one case. Additionally, the incidence of appendicitis in AN in the current study might be higher than that in the normal population.
\end{abstract}

Conclusions: These findings suggest that appendicitis should be considered as one of the potentially important complications in the therapy for AN.

Keywords: Appendicitis, Anorexia nervosa, Re-nourishment, Inpatient therapy

\section{Background}

Acute appendicitis is one of the common abdominal diseases and a variety of causes have been reported, including mechanical obstructions caused by fecalith [1], insufficient dietary fiber levels [2], and familial factors [3]. However, there have been no reports concerning the occurrence of appendicitis in patients with anorexia nervosa (AN) during inpatient care.

$\mathrm{AN}$ is a chronic and serious disease that presents not only with psychological but also physical symptoms and is accompanied by various physical complications [4-6]. Therefore, careful psychosomatic interventions are mandated [7]. During the re-nourishment period, severe physical complications, such as re-feeding syndrome, have to be taken into account; therefore, treatment strategies for AN need to be considered carefully, such as gradual increase of energy intake [8].

\footnotetext{
* Correspondence: shu-t@cephal.med.kyushu-u.ac.jp

'Department of Psychosomatic Medicine, Kyushu University Hospital, 3-1-1

Maidashi, Higashi-ku, Fukuoka-shi, Fukuoka 812-8582, Japan

Full list of author information is available at the end of the article
}

Here, we report three cases of appendicitis in patients with AN during re-nourishment period under inpatient care.

\section{Case presentation}

\section{Case 1}

This 34-year-old female patient had a fifteen-year history of AN and an eleven-year history of purging behavior such as vomiting after binging and abuse of large amounts of laxative. The patient had regularly consumed 20 tablets/ day of irritating laxatives until just before admission to our inpatient treatment unit. The patient's body mass index (BMI) was $13.6 \mathrm{~kg} / \mathrm{m}^{2}$. Table shows a summary of the patient's profile on admission. We started oral energy intake with $1600 \mathrm{kcal} /$ day from day 1 of admission, and the patient was able to consume the prescribed daily dose. We further prescribed a nonirritant laxative at a conventional dose to substitute the irritant laxatives. Although severe constipation improved, mild symptoms remained. On day 24 (BMI $\left.13.6 \mathrm{~kg} / \mathrm{m}^{2}\right)$, the patient manifested algor and high fever. In the physical examinations, few signs other than very mild abdominal tenderness 
around McBurney's point were detected. Leukocytes and $\mathrm{C}$-reactive protein (CRP) values in the blood test were elevated to $21740 / \mu \mathrm{l}$ and $2.48 \mathrm{mg} / \mathrm{dl}$, respectively (Table 1). Abdominal ultrasonography (US) could not detect any abnormality but abdominal computed tomography (CT) showed appendiceal enlargement suggesting acute appendicitis (Fig. 1a). Emergency surgery was performed at the Department of Surgery on the same day. Based on the post-operative pathological examination, the patient was diagnosed with acute phlegmonous appendicitis.

\section{Case 2}

This 17-year-old female patient had a 2-year history of AN without purging behaviors. The patient's BMI was $12.9 \mathrm{~kg} / \mathrm{m}^{2}$ on admission (Table 1). We started oral energy intake with $1200 \mathrm{kcal} /$ day and intravenous nutrition with $200 \mathrm{kcal} /$ day. We started a nonirritant laxative

Table 1 Summary of patients' profile and data

\begin{tabular}{|c|c|c|c|c|}
\hline & Case 1 & Case 2 & Case 3 & $\begin{array}{l}\text { Reference } \\
\text { values }\end{array}$ \\
\hline \multicolumn{5}{|l|}{ Patients' profile on admission } \\
\hline Age (year) & 34 & 17 & 38 & - \\
\hline Diagnosis & AN-BP & $A N-R$ & AN-BP & - \\
\hline BMI $\left(\mathrm{kg} / \mathrm{m}^{2}\right)$ & 13.6 & 12.9 & 11.9 & - \\
\hline \multicolumn{5}{|l|}{ EDI on admission } \\
\hline Total & 101 & 108 & 72 & - \\
\hline Drive for thinness & 3 & 19 & 18 & - \\
\hline Lack of interoceptive awareness & 22 & 16 & 22 & - \\
\hline Bulimia & 13 & 12 & 4 & - \\
\hline Body dissatisfaction & 11 & 22 & 8 & - \\
\hline Ineffectiveness & 24 & 19 & 6 & - \\
\hline Perfectionism & 8 & 4 & 9 & - \\
\hline Interpersonal distrust & 9 & 9 & 5 & - \\
\hline Maturity fears & 11 & 7 & 0 & - \\
\hline \multicolumn{5}{|l|}{ Laboratory data at onset } \\
\hline White blood cell $(/ \mu \mathrm{l})$ & 21400 & 12890 & 8240 & 3500-9000 \\
\hline Neutrophil (\%) & 89.7 & 91.5 & 86.8 & $40-70$ \\
\hline Red blood cell $\left(\times 10^{4} / \mu l\right)$ & 406 & 383 & 390 & $385-465$ \\
\hline Hemoglobin (g/dl) & 12.6 & 12.2 & 11.9 & $12.0-16.0$ \\
\hline AST (unit/l) & 22 & 33 & 38 & $13-33$ \\
\hline ALT (unit/l) & 20 & 79 & 49 & $6-30$ \\
\hline BUN (mg/dl) & 7 & 12 & 13 & $8-22$ \\
\hline $\mathrm{Cr}(\mathrm{mg} / \mathrm{dl})$ & 0.67 & 0.65 & 0.7 & $0.40-0.70$ \\
\hline CRP (mg/dl) & 2.48 & 1.20 & 0.09 & $\geqq 0.10$ \\
\hline
\end{tabular}

$B M I$ Body mass index, $E D I$ eating disorder inventory, $A N-B P$ anorexia nervosa binge-eating/purging type, $A N-R$ anorexia nervosa restricting type, $A S T$ Aspartate aminotransferase, ALT Alanine aminotransferase, $B U N$ blood urea nitrogen, Cr creatinine, CRP C-reactive protein
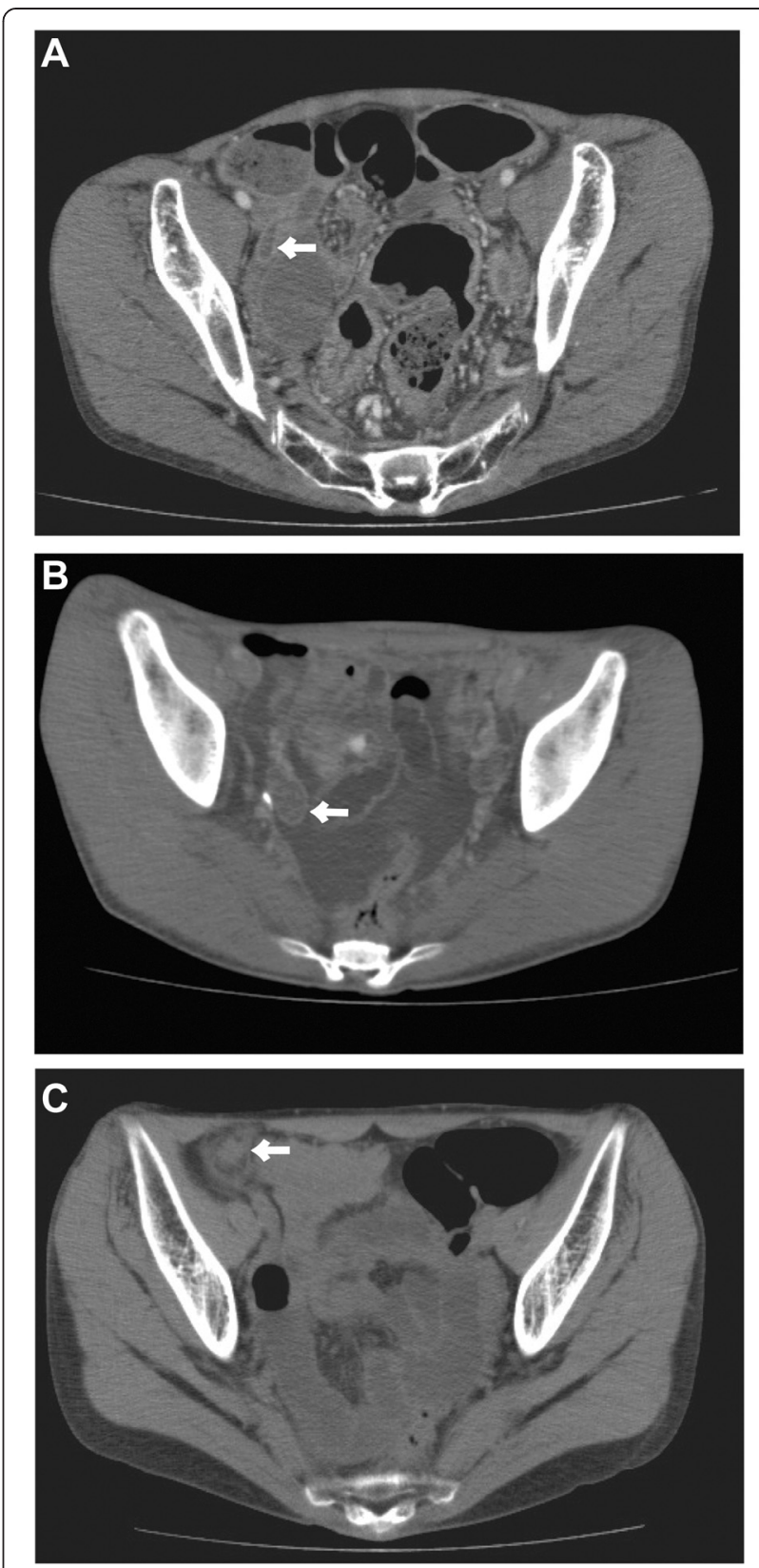

Fig. 1 Images of abdominal CT. a Case 1, b Case 2, c Case 3. Arrow indicates swollen appendix

at a conventional dose because of the patient's report of constipation. On day 4 (BMI $12.9 \mathrm{~kg} / \mathrm{m}^{2}$ ), the patient manifested algor, high fever, and diarrhea. In the physical examinations, very mild abdominal tenderness and increased bowel sound were detected. The leukocyte levels were elevated to $12890 / \mu \mathrm{l}$ and neutrophilia was observed (Table 1). Therefore, we first diagnosed her with acute enterocolitis and immediately started intravenous antibiotics therapy. However, high fever and abdominal pain persisted in spite of the five-day antibiotics therapy. 
Abdominal US could not detect any abnormality but abdominal CT (Fig. 1b) indicated an intrapelvic abscess on the right side and surgical operation was performed on the next day at the Department of Surgery. The patient was diagnosed with acute gangrenous appendicitis and localized peritonitis intraoperatively.

\section{Case 3}

This 38-year old female patient had an 8-year history of AN with purging behavior such as vomiting after binge eating and laxative abuse. The patient's BMI was $11.95 \mathrm{~kg} / \mathrm{m}^{2}$ on admission (Table 1). Pancytopenia was observed in the blood test on admission. We started a nonirritant laxative at a conventional dose because of the patient's report of constipation after admission; however, transient constipation was observed during the entire inpatient period. The patient's body weight gradually increased with inpatient therapy. On day 224 (BMI $16.2 \mathrm{~kg} / \mathrm{m}^{2}$ ), the patient manifested slight abdominal pain without muscular defense. Pyrexia was not observed and leukocyte values were within normal range (Table 1). CRP level was slightly elevated. Abdominal US could not detect any abnormality but abdominal CT (Fig. 1c) indicated acute appendicitis and surgical operation was performed on the same day at the Department of Surgery. Based on the post-operative pathological examination, the patient was diagnosed with acute phlegmonous appendicitis.

Treatment of AN in all three cases was continued following the successful surgical operations.

In a general year, more than a hundred new patients with eating disorder visit our clinic, and around $30 \mathrm{AN}$ patients are admitted to our inpatient unit. Over a period of recent 3 years, we observed three cases of appendicitis among the inpatients with AN (about $3 \%$ ). A report indicated that the lifetime prevalence of appendicitis was $8.6 \%$ in males and $6.7 \%$ in females, and the annual incidence of appendectomy was 11.8 per 10,000 population $(0.118 \%)$ in the United States [9]. According to the authors, the highest rate of incidental appendectomy was observed in females aged 35-44 years (43.8 per 10,000 population, $0.438 \%$ ), and the rate of appendicitis was found to be higher in Caucasians than in non-Caucasians. Because our study was retrospective and was confined to a small set of cases (i.e., only three) that had been referred to us -rather than having been selected using methods to guarantee statistically representative appendicitis caseswe are cautious about making inferences regarding the incidence or the prevalence of appendicitis associated with AN care. Thus, the incidence of appendicitis during AN treatment in the current study might be higher than that in the general population. Furthermore, although the possibility remains that it was incidental in our cases, appendicitis in all three cases occurred during the renourishment period while being hospitalized for therapy.
These observations suggest that we should consider appendicitis as one of the gastrointestinal complications of AN under re-nourishment condition.

Gastrointestinal complications, such as constipation, due to chronically abnormal eating behaviors or use of irritant laxatives, are often observed and have been reported among AN patients [10, 11]. However, there have been no reports on the occurrence of appendicitis during the treatment of AN.

Acute appendicitis is one of the common abdominal diseases and a variety of causes have been reported; for example, mechanical obstruction represents one of the causes of appendicitis $[1,12]$. Therefore, chronic constipation might be related to occurrence of appendicitis. A tendency for constipation, at varying degrees, was observed in all three cases that showed different AN subtypes. Constipation resulting from inadequate food intake is one of the common complications of AN. Chiarioni reported that most anorexic women complaining of constipation had slow colonic transit times [10]. In addition, among the binge eating/purging type of AN patients, cathartic colon syndrome [13] induced by chronic use of irritant laxatives might be the precipitating cause of constipation. Furthermore, large amounts of stool, observed in the abdominal CT, probably resulted from a combination of adequate food intake and constipation tendency under inpatient condition. To prevent complications of appendicitis, careful control of defecation might be important during the re-nourishment period under inpatient care in AN patients.

The development of appendicitis in Case 3 occurred on the $224^{\text {th }}$ day, while appendicitis development in Case 1 and Case 2 occurred during a relatively early stage of re-nourishment. The underlying cause of this difference in the timing of appendicitis onset is not clear; however, in Case 3, latent constipation may have been a pre-existing chronic condition.

Appropriate diagnosis and treatment is most important to prevent the risk of perforation or peritonitis. None of the three cases manifested severe abdominal symptoms observed in appendicitis such as Rovsing's sign or Blumberg sign. Especially in case 2, the clinical condition progressed to gangrenous appendicitis and localized peritonitis, as first manifestations had been diffuse and seemed to indicate acute enterocolitis and correct diagnosis was delayed. In case 3, leukocyte level was within normal range, and CRP level was only slightly elevated, probably because pancytopenia had persisted.

To diagnose appendicitis, abdominal US is generally useful and can be the first imaging method of choice $[14,15]$. However, in all three cases, abdominal US had difficulty in detecting abnormality. Therefore, we also performed abdominal $\mathrm{CT}$, which proved to be more helpful for diagnosis. 
In general, it is more difficult to make a diagnosis of appendicitis if the patient is pregnant [16] or a child [17]. Salo et al. [18] reported that diffuse symptoms in younger children with appendicitis led to delayed diagnosis and complicated appendicitis. Because interoceptive impairments are often reported in the eating disorder inventory [19] among AN patients [20], their perception of bodily signals might be reduced. Thus, similar to the report by Salo et al., mild and diffuse symptoms were observed in the AN cases described above.

\section{Conclusion}

Patients with AN may exhibit diffuse and mild symptoms of appendicitis resulting in difficulties and delays in diagnosis. To prevent appendicitis under re-nourishment during the treatment of $\mathrm{AN}$, careful control of defecation might be important. Though the present study is an observation that there may be a relationship between the cases of appendicitis and AN re-nourishment and is not conclusive proof of cause, these findings suggest that appendicitis should be considered as one of the potentially important complications in the therapy for AN.

\section{Consent}

Oral informed consent was obtained from the patients for publication of this case report and accompanying images.

\section{Abbreviations}

AN: anorexia nervosa; AN-BP: anorexia nervosa, binge eating/purging type; AN-R: anorexia nervosa, restricting type; BMI: body mass index; CRP: C-reactive protein; CT: computed tomography; EDI: eating disorder inventory; US: ultrasonography.

\section{Competing interests}

Shu Takakura, Hiroaki Yokoyama, Chie Suzuyama, Keita Tatsushima, Makoto Yamashita, Motoharu Gondou, Chihiro Morita, Tomokazu Hata, Masato Takii, Keisuke Kawai, and Nobuyuki Sudo declare no conflicts of interests.

\section{Authors' contributions}

Shu Takakura participated in the therapy for the patients, the conceptualisation of the study, the data collection, and the writing of the paper. Hiroaki Yokoyama, Chie Suzuyama, Keita Tatsushima, Makoto Yamashita, Motoharu Gondou, Chihiro Morita, Tomokazu Hata, Masato Takii, Keisuke Kawai, and Nobuyuki Sudo participated therapy for the patients and editing of the manuscript. All authors read and approved the final manuscript.

\section{Acknowledgements}

Authors would like to thank the members of the Department of Surgery and Oncology and the Department of Surgery and Science of Kyushu University Hospital for successful operation of the patients.

This work was supported in part by JSPS KAKENHI Grant Number 15 K08920.

\section{Author details}

'Department of Psychosomatic Medicine, Kyushu University Hospital, 3-1-1 Maidashi, Higashi-ku, Fukuoka-shi, Fukuoka 812-8582, Japan. ²Department of Psychosomatic Medicine, Graduate School of Medical Sciences, Kyushu University, 3-1-1 Maidashi, Higashi-ku, Fukuoka-shi, Fukuoka 812-8582, Japan.

\section{References}

1. Ramdass MJ, Young Sing Q, Milne D, Mooteeram J, Barrow S. Association between the appendix and the fecalith in adults. Can J Surg J Can Chir. 2014;57(6):002014-2014. doi:10.1503/cjs.002014.

2. Barker DJ. Acute appendicitis and dietary fibre: an alternative hypothesis Br Med J (Clin Res Ed). 1985;290(6475):1125-7.

3. Drescher MJ, Marcotte S, Grant R, Staff L. Family history is a predictor for appendicitis in adults in the emergency department. West J Emerg Med. 2012;13(6):468-71. doi:10.5811/westjem.2011.6.6679.

4. Kawai K, Yamashita S, Komaki G, Shimizu M, Nakashima M, Etou S, et al. The outcome of treatment for anorexia nervosa inpatients who required urgent hospitalization. BioPsycho Soc Med. 2014;8:20. doi:10.1186/1751-0759-8-20.

5. Takakura S, Nozaki T, Nomura Y, Koreeda C, Urabe H, Kawai K, et al. Factors related to renal dysfunction in patients with anorexia nervosa. Eating Weight Disorders EWD. 2006;11(2):73-7.

6. Zipfel S, Lowe B, Reas DL, Deter HC, Herzog W. Long-term prognosis in anorexia nervosa: lessons from a 21-year follow-up study. Lancet. 2000;355(9205):721-2. doi:10.1016/S0140-6736(99)05363-5.

7. Amemiya N, Takii M, Hata T, Morita C, Takakura S, Oshikiri K, et al. The outcome of Japanese anorexia nervosa patients treated with an inpatient therapy in an internal medicine unit. Eating Weight Disord EWD. 2012;17(1):e1-8. doi:10.3275/8034

8. Treasure J, Claudino AM, Zucker N. Eating disorders. Lancet. 2010:375(9714):583-93. doi:10.1016/S0140-6736(09)61748-7.

9. Addiss DG, Shaffer N, Fowler BS, Tauxe RV. The epidemiology of appendicitis and appendectomy in the United States. Am J Epidemiol. 1990;132(5):910-25.

10. Chiarioni G, Bassotti G, Monsignori A, Menegotti M, Salandini L, Di Matteo G et al. Anorectal dysfunction in constipated women with anorexia nervosa. Mayo Clin Proc. 2000;75(10):1015-9. doi:10.4065/75.10.1015.

11. Chun AB, Sokol MS, Kaye WH, Hutson WR, Wald A. Colonic and anorectal function in constipated patients with anorexia nervosa. Am J Gastroenterol. 1997;92(10):1879-83.

12. Jones BA, Demetriades D, Segal I, Burkitt DP. The prevalence of appendiceal fecaliths in patients with and without appendicitis. A comparative study from Canada and South Africa. Ann Surg. 1985;202(1):80-2.

13. Joo JS, Ehrenpreis ED, Gonzalez L, Kaye M, Breno S, Wexner SD, et al. Alterations in colonic anatomy induced by chronic stimulant laxatives: the cathartic colon revisited. J Clin Gastroenterol. 1998;26(4):283-6.

14. Gaitini D, Beck-Razi N, Mor-Yosef D, Fischer D, Ben Itzhak O, Krausz MM, et al. Diagnosing acute appendicitis in adults: accuracy of color Doppler sonography and MDCT compared with surgery and clinical follow-up. AJR Am J Roentgenol. 2008;190(5):1300-6. doi:10.2214/AJR.07.2955.

15. Mallin M, Craven P, Ockerse P, Steenblik J, Forbes B, Boehm K, et al. Diagnosis of appendicitis by bedside ultrasound in the ED: a case series. Am J Emerg Med. 2014. doi:10.1016/j.ajem.2014.10.004

16. Andersen B, Nielsen TF. Appendicitis in pregnancy: diagnosis, management and complications. Acta Obstet Gynecol Scand. 1999:78(9):758-62.

17. Smink DS, Finkelstein JA, Kleinman K, Fishman SJ. The effect of hospital volume of pediatric appendectomies on the misdiagnosis of appendicitis in children. Pediatrics. 2004;113(1 Pt 1):18-23.

18. Salo M, Friman $G$, Stenstrom $P$, Ohlsson B, Arnbjornsson E. Appendicitis in children: evaluation of the pediatric appendicitis score in younger and older children. Surg Res Practice. 2014;2014:438076. doi:10.1155/2014/438076.

19. Garner DM, Olmsted MP. Scoring the eating disorder inventory. Am J Psychiatry. 1986;143(5):680-1.

20. Khalsa SS, Craske MG, Li W, Vangala S, Strober M, Feusner JD. Altered interoceptive awareness in anorexia nervosa: Effects of meal anticipation, consumption and bodily arousal. Int J Eating Disord. 2015. doi:10.1002/eat.22387. 